\title{
The Role of Escherichia coli in the Development and Progression of Cancer
}

\author{
Marco Antonio Hernández-Luna ${ }^{1 *}$, Hugo Enrique Lagunes-Servin ${ }^{2}$, \\ Sergio Lopez-Briones ${ }^{1}$
}

\footnotetext{
${ }^{1}$ Departamento de Medicina y Nutrición, División Ciencias de la Salud, Universidad de Guanajuato, Campus León. Guanajuato, México.

${ }^{2}$ Unidad de Investigación en Salud, Secretaria de Salud de Aguascalientes. Aguascalientes, México

${ }^{1 *}$ marco.hernandez@ugto.mx
}

\begin{abstract}
Escherichia coli (E. coli) is a widely distributed microorganism in nature and normally colonize the human gastrointestinal tract. E. coli strains are part of the normal gut microbiota. In recent years, some E. coli strains producing the genotoxin colibactin have been associated with development and progression of colon cancer. However, the role of E. coli and their toxins in carcinogenesis is not entirely clear. Thus, some proteins secreted by bacteria may trigger and promote tumor development. In this paper, we focus on reviewing some proteins produced by E. coli, which have been associated with cancer. Also possible mechanisms involved on transformation of epithelial cells into tumor cells, are briefly described.
\end{abstract}

Keywords: Escherichia coli, colon cancer, prostate cancer, pks island.

Abbreviations: $E$. coli $=$ Escherichia coli, $P D G F=$ platelet-derived growth factor, $C D=C r o h n ' s$ disease, AIEC = Adherent-Invasive E. coli, CEACAM6= Carcinoembryonic antigen related cell adhesion molecule 6 , $C D T=$ Cytolethal distending toxin, Cif = Cycle inhibiting factor, CNF1=Cytotoxic Necrotizing Factor, EPEC= Enteropathogenic Escherichia coli, EHEC= Enterohemorrhagic Escherichia coli, UPEC= Uropathogenic Escherichia. coli, hly= $\alpha$-hemolysin, HIF-1 $\alpha=$ Hypoxia Inducible Factor-1 $\alpha$, VEGF= Vascular Endothelial Growth Factor, MIC-1= Macrophage Inhibitory Citokine 1, EGFR= Epidermal Growth Factor Receptor, EGF = Epidermal growth factor receptor.

\section{INTRODUCTION}

Escherichia coli (E. coli) is a Gram-negative bacterium, commonly found as normal flora in the intestines of human, and another animal species. In addition, it is one of the most studied microorganisms worldwide.

Phylogenetically it has been classified into 5 groups (A, B1, B2, D, and E) (1). The phylogenetic distribution of these $E$. coli strains change depending of both the region and human population they colonize (2). In recent years, interest on E. coli has increased because E. coli colonization of the colon has been reported as a risk factor to development colorectal cancer (3).

In this paper, we focus on reviewing the proteins and possible mechanisms, through E. coli could promote the development and progression of colon cancer, as well as cancer in genitourinary tract.

\section{Microbiota and Inflammation}

Intestinal microbiota including commensal strains of $E$. coli have an important role in the homeostasis of the gastrointestinal tract (4). However, when dysbiosis take place, an imbalance on the normal microbial population into intestinal compartment is produced. Therefore, most beneficial bacteria are damaged, favoring the overgrowth of those pathogenic bacteria (5); altering the physiology and function in intestinal compartment, as well as promoting the development of intestinal diseases such as Crohn's disease and colon cancer. Dysbiosis may be induced by antibiotic exposure, diet and other factors. The local intestinal inflammation is an important factor to trigger dysbiosis (6).

In general, inflammation has been established as a risk factor for the development of several cancers including colon cancer; because different cytokines and chemokines may promote the development and progression of tumors (7). In colon cancer, alterations in cytokines pattern production have been detected (8), with increased levels of IL-8 (9), IL-6 (10), and the platelet-derived growth factor 
(PDGF) (11). In addition, high levels of IL-6 and VEGF have been proposed as prognostic markers in colon cancer $(10,12)$.

On the other hand, chronic inflammation is a source of molecules associated to either DNA induceddamage or inhibiting DNA repair mechanisms (13), mainly by high production of reactive oxygen species (14). Also, the presence of these chronic inflammatory stimuli may turn off the expression of tumor suppressor genes $(15,16)$ and stimulate the production of angiogenic factors $(17,18)$.

The origin of chronic inflammation has been associated to bacterial and virus infections, such as Helicobacter pylori (19), and papillomavirus (20). Nevertheless, the precise mechanism by which chronic inflammation triggers the development of cancer is not entirely clear; but it has been suggested that inflammation is not sufficient to trigger a cancer event and bacteria are required. In this regard, bacterial agents promote the carcinogenic processes through effector proteins, which induce DNA damage in the host cell (21).

\section{Escherichia coli and Colon Cancer}

Some E. coli strains have been associated with inflammatory diseases of the gastrointestinal tract such as Crohn's disease (CD), where bacteria colonize the intestinal mucosa promoting an inflammatory process (22) and subsequently may trigger the development of colon cancer. Interestingly, a low percentage of patients with colon cancer, suffered an inflammatory bowel disease earlier. However, it is considered a high-risk factor for cancer development (23).

The interest to investigate $E$. coli in colon cancer began nearly two decades ago, when it was reported the presence of bacteria in colon cancer biopsies (24). Since that discovery, various studies have focused on figure out the role of $E$. coli in development and progression of colon cancer, where the chronic inflammation could be associated with dysbiosis in the gastrointestinal tract, allowing $E$. coli strains overgrowth, and it could lead to alteration of the intestinal epithelial cells (25).

The mainly E. coli strains associated with $\mathrm{CD}$ and colon cancer are member of the phylogenetic group B2. In this regard, the LF82 E. coli strain has been found in biopsies from patients with colon cancer (3). An important characteristic of LF82 strain is the capability to attach and invade the intestinal epithelium cells, and it has been named Adherent-Invasive E. coli or AIEC (26). Moreover, it was reported that adherence of AIEC to the intestinal epithelium is through the CEACAM6 receptor (carcinoembryonic antigen related cell adhesion molecule 6) (27), which is over expressed on the intestinal epithelium cells from both Crohn's disease and colon cancer patients (28). Likewise, over expression of CEACAM6 has been associated with intestine inflammation, and this mechanism is dependent of IL-6 production (29). For this reason, it is possibly than inflammatory process not only favor the overgrowth of $E$. coli but also increasing their invasiveness through over expression of CEACAM6 receptor, and this would be a first step for malignant transformation of colon cells, and after of infection the next step would be the alteration of DNA in host cell through of bacterial toxins such as cyclomodulins.

\section{Cyclomodulins and Cancer}

The term cyclomodulin was proposed in 2005 by Nougayrede J.P. et al. (30) to describe those bacterial toxins able to regulate the cell cycle by either inducing or promoting the cellular arrest. For several years, some research groups have been focused on studying the role of these toxins, as well as their mechanisms involved in cancer development; because increased production of cyclomodulins by E. coli from the phylogenetic group B2, have been detected in biopsies from colon cancer patients (3).

The most common cyclomodulins are the following:

1. Cytolethal Distending toxin or CDT, produced by several bacteria strains including both $E$. coli and Salmonella typhi (S. typhi). An important mechanism triggered by CDT is to block the cell cycle between the $\mathrm{G} 2$ and mitosis phases (31).

2. Cycle Inhibiting Factor or Cif is produced by pathogenic strains such as Entero Pathogenic $E$. coli (EPEC) and Entero Hemorrhagic E. coli (EHEC). Among the multiple effects induced by cif are the nuclear elongation and starting the DNA synthesis in infected cells, independently of cell division (32).

3. Cytotoxic Necrotizing Factor 1 or CNF1 is produced by Uropathogenic E. coli strain (UPEC) and induces the GTPases activation, promoting both gene transcription and cell proliferation (33). 
4. Colibactin is expressed by E. coli strains belonging to the intestinal microbiota and they have been associated with DNA damage (34).

To date, the role of CDT, Cif and CNF1 in development of cancer is not completely clear. Although, recently it has been reported that CDT induces malignant transformation of HCECs epithelial cells (35). On the other hand, colibactin is a ciclomodulin involved in development of colon cancer, because inflammation induces its expression througth $p k s$ pathogenicity island activation.

\section{pks island and Colibactin}

The pks pathogenicity island is a genes group highly conserved in the family of Enterobacteriaceae, including Enterobacter aerogenes, Citrobacter koseri, Klebsiella pneumonia and Escherichia coli (36-39). Interestingly, the pks island is present only in the E. coli strains from intestinal microbiota and extra-intestinal E. coli (ExPEC), but not in E. coli pathogenic strains such as EPEC and EHEC (36).

The regulation of pks island by inflammation still remains to be elucidated. However, using colitis murine models has allowed to known the role of pks island on development of colon cancer (25). In this regard, E. coli overgrowth was originated by intestinal dysbiosis and consequently the development of colon cancer in IL-10 -/- knockout (KO) mice after azoxymethane induced inflammation (25). Likewise, IL-10 -/- KO mice infected with pks island -/- E. coli mutant strain developed colon cancer, but in a lesser extent than $1 \mathrm{~L}-10$-/- KO mice infected with E. coli WT strain (25). Moreover, progression of gut inflammation modifies the $E$. coli. transcriptome favoring the $p k s$ island expression (40). All these data suggest that E. coli is required to promote the development of cancer, and inflammation would be important to triggering the production of genotoxic proteins by bacteria. The mechanisms by which $E$. coli strains use different proteins to regulate inflammation in the host cells has been reviewed by some researchers (41-44). So far, mechanisms about regulation of pks island, as well as colibactin production have not been described entirely, but it could be a bacteria adaptation mechanism to the inflammatory microenvironment.

Thereby, it is possible that synergistic action between both the inflammatory process in intestine and $p k s+E$. coli strains, such as LF82 are required to induce colon cancer. Recent findings support this idea, because an increased tumor progression in murine xenograft model was observed after infection with $p k s+E$. coli strains compared with those infected by pks - strains $(45,46)$. Although the mechanism by which $p k s+E$. coli strains induced tumor progression is not completely clear, it was reported that infection with pks $+E$. coli strains produced DNA damage, cell cycle arrest, melanocytosis, aneuploidy and tetraploidy, thus all these effects could be associated with DNA mutations, and accordingly induce cancer $(38,47)$. On the other hand, activation of $p k s$ island induces miR-20a expression, a microRNA associated with control of senescence in intestinal epithelial cells through downregulation of sumo specific protease 1 protein or SENP-1 (45). The SENP-1 controls the process of cellular senescence via SUMOylation of transcriptor factor p53 (48) (Figure 1).

Although most studies have focused on the complete pks island, an important product encoded inside pks island is the colibactin toxin; a secondary metabolite of a Non-Ribosomal Peptide Synthasepolyketide synthase (NRPS-PKS) (34). Recently, the colibactin biosynthesis pathway started to be elucidated (49), which highly support the hypothesis that colibactin induce DNA damage. Moreover, it was proposed that colibactin directly alkylates the DNA in the host cell (50). These data indicated that colibactin is essential for genotoxicity and cellular transformation. Interestingly, within the $p k s$ island genes there is also a gen sequence encoding for CLBs protein, which the main role is colibactin function inhibition (51). Likewise, a recent study suggests that colibactin is not involved on development and maintenance of inflammation. In contrast, the macrophages infiltration into the intestinal epithelium is induced by overgrowth of $p k s+E$. coli strains, and inflammation dependent on COX-2 activation is established (52). Thus, once established the intestinal inflammation, E. coli proliferation is increased and the production of colibactin started after pks island activation. Therefore, it possible that above mechanisms would be associated with the development of colon cancer.

Figure1. Alteration of Intestinal Epithelial Cells Caused by the Inflammatory Process Favor AIEC Proliferation and Malignancies Cell Transformation.

Under normal bowel conditions commensal E. coli colonize intestinal epithelium, there is a low expression of CEACAM6, and there is not activation of pks island. Nevertheless, during 
inflammation, the intestine dysbiosis favors the growth of AIEC and induces activation of the pks island by unknown mechanism. In adddition, the increase of IL- 6 due to the inflammatory process induces CEACAM6 expression increasing invasiveness of AIEC. Once AIEC has been internalized in the epithelial cell, colibactin is produced by the bacteria may cause DNA damage. And could be associated with cellular senescence through activation of miR-20a-5p which down-regulate SENP-1, increasing the SUMOylation of $\mathrm{p} 53$.

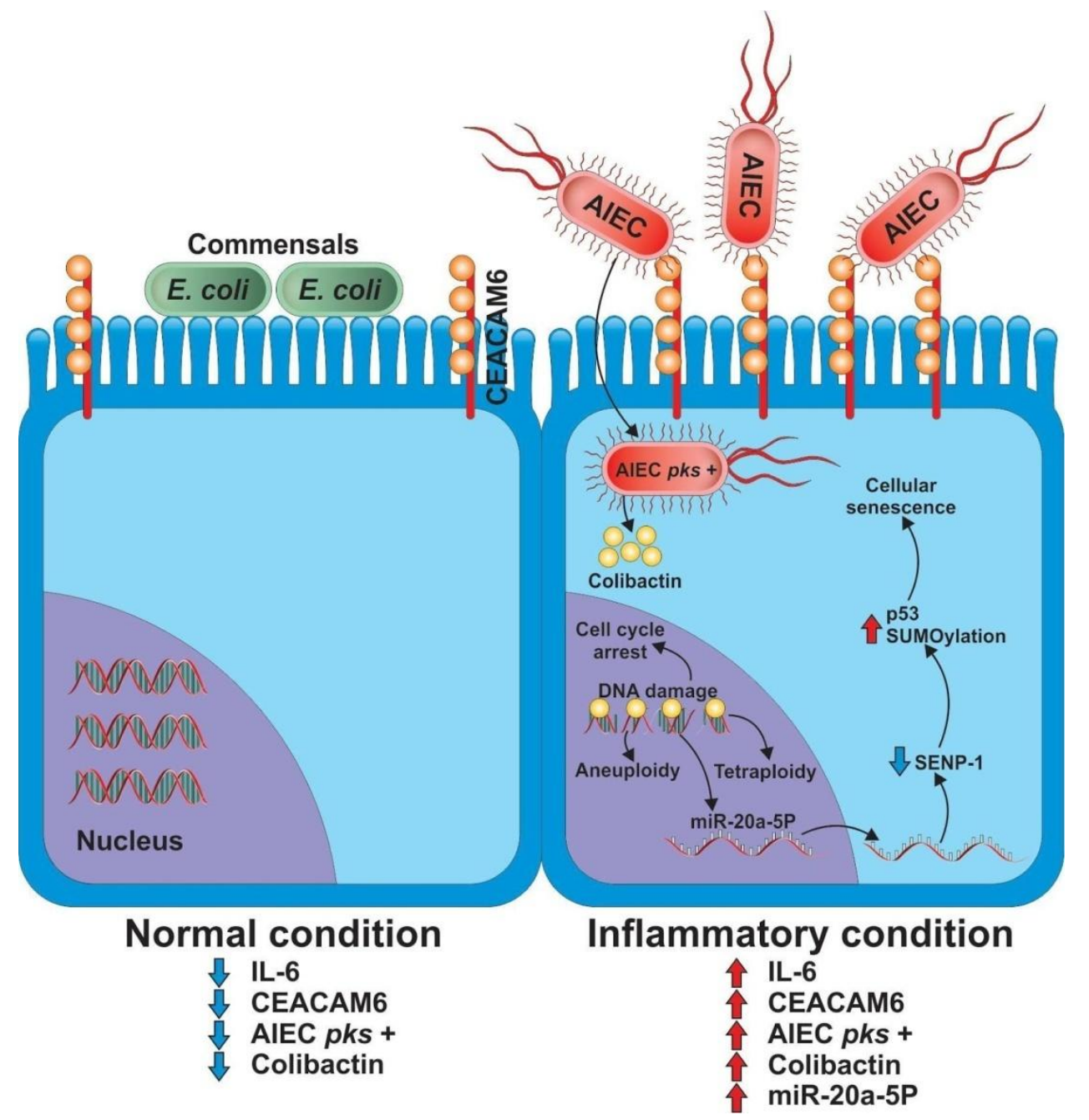

\section{a-hemolysin and Colon Cancer}

As mentioned above, different studies have suggested that development and progression of colon cancer are associated mainly to dysbiosis; where most beneficial bacteria are damaged and overgrowth of AIEC is produced; as well as activation of pks island and colibactin production. However, some, studies suggest that other E. coli pathogenic strains such as EPEC and EHEC are involved $(53,54)$. Therefore the actions of some other $E$. coli effector proteins could also be involved in the development of colon cancer; such as $\alpha$-hemolysin (hly), because E. coli strains hly + have been detected in samples from colon cancer patients (53). Although the role of hly $+E$. coli strains in colon cancer is unclear, it has been suggested that hly may increase the expression of GLUT 1 receptor (Glucose transporter 1), as well as decrease the expression of tumor suppressor protein BIM, through regulation of transcription factor HIF-1 $\alpha$ (Hypoxia Inducible factor $1 \alpha$ ) (Figure 2). However, it is not completely clear whether regulation of HIF-1 $\alpha$ expression is a direct effect of hly, because $E$. coli LPS in vitro stimulation induced accumulation of HIF-1 $\alpha$ in fibroblasts, as well as increased levels of VEGF mRNA (55). In addition, when a colon cancer cell line was infected with LF82 E. coli strain, an important increase of both HIF-1 $\alpha$ and VEGF was observed (56). Therefore, up-regulation of HIF-1 $\alpha$ and VEGF by $E$. coli infection could be an early event in colon carcinogenesis. Moreover, high expression of HIF- $1 \alpha$ is induced by hypoxic microenvironment $(57,58)$. Leading to down regulation of BIM (58). Interestingly, increased expression of HIF-1 $\alpha$ induce up-regulation of CEACAM6 receptor; facilitating a secondary infection by E. coli, and increasing the E. coli survival inside the cell (56). 
Figure 2. Cellular Alteration by Pathogenics Escherichia Coli.

Infection of the intestinal epithelium by EHEC will participate in cancer progression through effectors such as $\alpha$-hemolysin, which modified the expression of BIM, GLUT 1, HIF-1 $\alpha$ and VEGF, all related to colon cancer. Furthermore, infection by EPEC could be associated with mutations in intestinal cells through down-regulate MLH1 and MSH2 proteins by unknown mechanism. In addition, the effectors EspF and EspZ have been associated with phosphorylation of EGFR, which is related with angiogenesis and cell survival. Additionally, EPEC could favor the process of metastasis through inducing secretion of MIC-1 from infected macrophages and EspF, which disrupt the tight junction proteins favoring the migration of tumor cells.

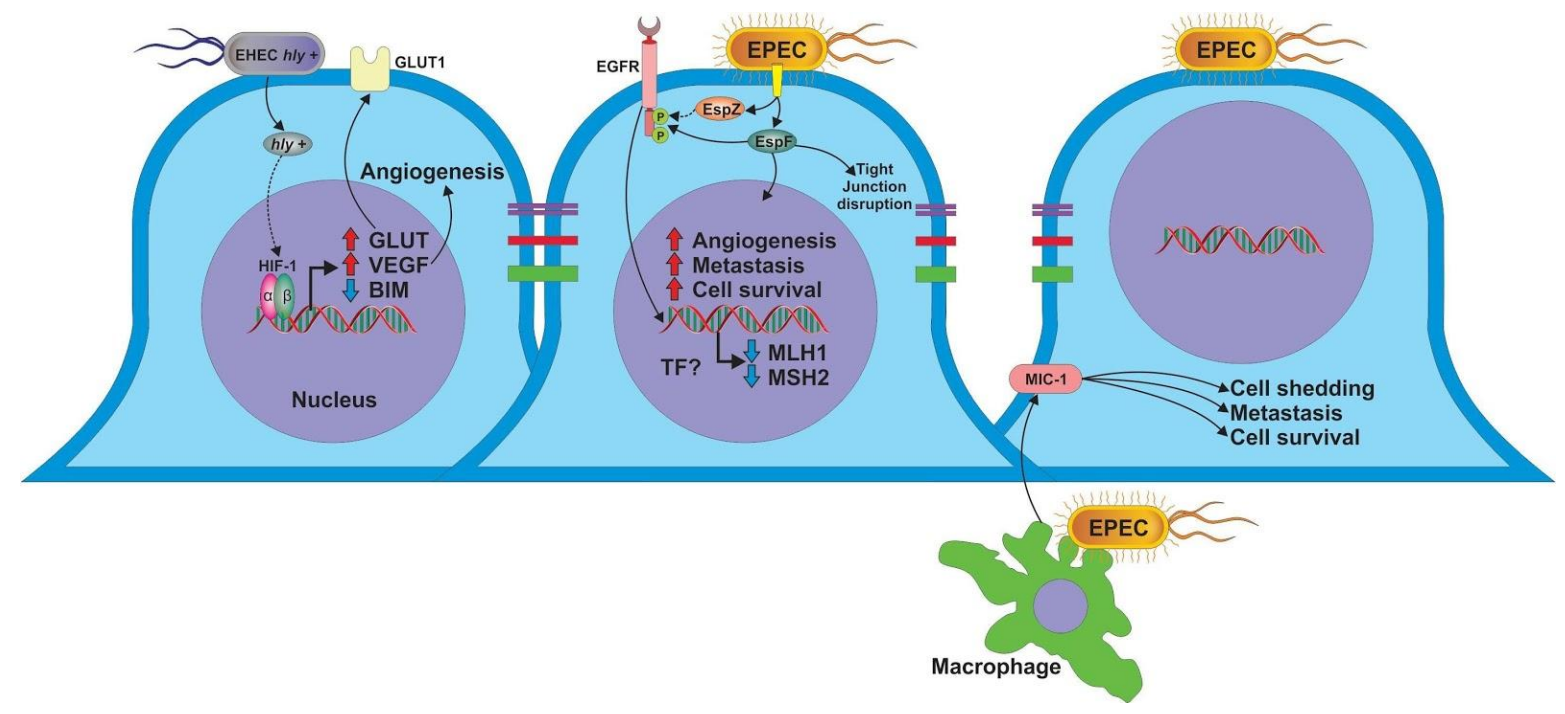

\section{Entero Pathogenic Escherichia coli and Colon Cancer}

As previously was mentioned, E. coli was found in a high percentage of biopsies from colon cancer patients (24) and the bacteria most common found were AIEC strains (3). However, Entero Pathogenic E. coli (EPEC) strains have been also detected in biopsies from colon cancer patients (54, 59). Although it is well known that EPEC is the main cause of diarrhea in children (60), the infection in adults is not completely clear. The presence of EPEC in biopsies from colon cancer patients could also be related to this neoplasm; because in vitro infection of colon cancer cell lines with EPEC causes depletion of proteins associated with DNA repair (mismatch repair MMR) such as MLH1 and MSH2, and consequently somatic mutations in the intestinal epithelial cells are produced (59). The mechanism by which EPEC infection induces DNA damage is not clear, but it has suggested that EspF protein is involved (61). EspF is an important effector protein expressed by both EPEC and EHEC (62), which is easily internalized by epithelial cell through the Type III Secretion System (63). However, it is unclear whether EspF acts directly on MLH1 and MSH2 or indirectly inducing activation of some transcription factors, which down-regulate MLH1 and MSH2 expression. This hypothesis is supported by previous in vitro and in silico assays, where some nuclear targets for $E$. coli proteins were described $(64,65)$. Hence, bacterial proteins could enter to cell nucleus and attach to target genes. This way important changes on host cell genes could be produced, causing DNA mutations and triggering colon carcinogenesis (66).

Additionally, EspF is capable to disrupt the tight junctions proteins in intestinal epithelial cells (67), which could facilitate the release and dissemination of tumor cells, and contributing to metastasis of colon cancer. In addition, EPEC infection increased the expression of macrophage inhibitory citokine 1 (MIC-1), this molecule has been associated with an important increase on survival and spread of colon tumor cells in a GTPase RhoA dependent pathway (68).

Also, EPEC infection induces the phosphorylation of EGFR receptor (69). This cellular mechanism is related to the development of colon cancer $(70,71)$, and up regulation of EGFR has been correlated with a poor prognosis in colon cancer patients (72). Although the mechanism by which EPEC induces EGFR phosphorylation is not entirely clear, it has been showed that translocation of effectors EspF and EspZ could be associated with EGFR phosphorylation (73). EPEC proteins and their possible mechanisms are summarized in Figure 2. 


\section{E. coli and Genitourinary Cancer}

Currently, E. coli is a bacterium that has acquired relevance in colon cancer research. Nevertheless, $E$. coli not only colonizes the gastrointestinal tract but also genitourinary tract; here bacteria could disrupt the epithelial barrier favoring the development of malignancies such as cancer bladder (74). Moreover, E. coli could promote prostate cancer, because has been observed that prostatic secretion of patients with this neoplasm have increased number bacteria, including E. coli; similary as it was determined in colon cancer biopsies (75).

Additionally, in murine model of prostate infection with Uropathogenic E. coli (UPEC), an acute inflammatory response, epithelial cell proliferation and reactive hyperplasia were produced (76). In addition, the expression of prostate-specific antigens (PSA), the principal markers of development of prostate cancer have been identified and developed (77).

UPEC is the main pathogenic agent associated with urinary tract infections (UTIs) (78). UPEC strains share some features on pathogenicity islands with those E. coli strains isolated from patients with Crohn's disease or colon cancer (79). Similarly, than AIEC, UPEC attach to CEACAM receptors to adhere to the epithelial cells. Nevertheless, the molecules of AIEC involved in the interaction with CEACAM have not been identified, meanwhile it is well known that Afa/Dr adhesin expressed on UPEC interact with CEACAM receptor (80).

In addition, it has been reported that UPEC infection induced methylation of the tumor suppressor gene $C D K N 2 A$ in uroepithelial cells (81). This mechanism has been associated with prostate cancer (82). Therefore, all these data suggest a possible role of UPEC in triggering and development of prostate cancer. For this reason, must to be important to study the role of E. coli on the development of genitourinary tract carcinogenesis.

\section{CONCLUSIONS}

The chronic inflammation is a major risk factor for developing cancer, recently it has been shown that the presence of infectious agents such as pathogenic bacteria, is also an important factor that participate in development of cancer by two ways: 1) contributing to the inflammatory process and 2) simulating the secretion of bacterial proteins, which modify the cellular function. The proliferation of E. coli during processes such as intestinal dysbiosis, and chronic inflammation, could contribute to the neoplastic process through the pks island activation. The $p k s$ island is expressed by both commensal E. coli strains, as well as pathogenic strains such as AIEC. Nevertheless, the regulation mechanisms of $p k s$ island still remain to be elucidated. Another important factor that may favor the development of cancer is the effector proteins such as $\alpha$-hemolysin and EspF produced and secreted by pathogenic strains such as EPEC and EHEC. They are involved in several mechanisms that regulate the development and progression of colon cancer. Moreover, several host proteins such as VEGF, EGFR, BIM and HIF-1 $\alpha$, are involved in colon cancer, but the precise molecular mechanisms triggered or regulated by $E$. coli proteins is still unknown.

The role of E. coli in genitourinary tract carcinogenesis is not entirely clear. Although, UPEC strains are capable to infect bladder and kidney, and they share genes with E. coli strains isolated from patients with colon cancer, it is unknown whether these genes are related to trigger and cancer development. Therefore, it will be interesting to study the relation between UPEC and genitourinary carcinogenesis, more studies are needed to establish the role of E. coli and its toxins in the development of cancer

Finally, to determine the specific role of E. coli, as well as their proteins in cancer development and progression further investigation need to be carrying out.

\section{ACKNOWLEDGEMENTS}

The authors thank the Directorate for Research Support and Postgraduate Programs at the University of Guanajuato for their support in the translation and editing of the English-language version of this article. We thank the Universidad de Guanajuato and Instituto de Servicios de Salud del Estado de Aguascalientes for the financial support.

\section{REFERENCE}

[1] Chaudhuri, R. R., and Henderson, I. R. (2012) The evolution of the Escherichia coli phylogeny. Infection, genetics and evolution: journal of molecular epidemiology and evolutionary genetics in infectious diseases 12, 214-226. 
[2] Tenaillon, O., Skurnik, D., Picard, B., and Denamur, E. (2010) The population genetics of commensal Escherichia coli. Nature reviews. Microbiology 8, 207-217.

[3] Buc, E., Dubois, D., Sauvanet, P., Raisch, J., Delmas, J., Darfeuille-Michaud, A., Pezet, D., and Bonnet, R. (2013) High prevalence of mucosa-associated E. coli producing cyclomodulin and genotoxin in colon cancer. PloS one 8, e56964.

[4] Leser, T. D., and Molbak, L. (2009) Better living through microbial action: the benefits of the mammalian gastrointestinal microbiota on the host. Environmental microbiology 11, 2194-2206.

[5] Hawrelak, J. A., and Myers, S. P. (2004) The causes of intestinal dysbiosis: a review. Alternative medicine review : a journal of clinical therapeutic 9, 180-197.

[6] Butto, L. F., and Haller, D. (2016) Dysbiosis in intestinal inflammation: Cause or consequence. International journal of medical microbiology: IJMM.

[7] Mager, L. F., Wasmer, M. H., Rau, T. T., and Krebs, P. (2016) Cytokine-Induced Modulation of Colorectal Cancer. Frontiers in oncology 6, 96.

[8] Cui, G., and Florholmen, J. (2008) Polarization of cytokine profile from Th1 into Th2 along colorectal adenoma-carcinoma sequence: implications for the biotherapeutic target? Inflammation \& allergy drug targets 7, 94-97.

[9] Ueda, T., Shimada, E., and Urakawa, T. (1994) Serum levels of cytokines in patients with colorectal cancer: possible involvement of interleukin-6 and interleukin-8 in hematogenous metastasis. Journal of gastroenterology 29, 423-429.

[10] Knupfer, H., and Preiss, R. (2010) Serum interleukin-6 levels in colorectal cancer patients--a summary of published results. International journal of colorectal disease 25, 135-140.

[11] Belizon, A., Balik, E., Horst, P. K., Shantha Kumara, H. M., Nasar, A., and Whelan, R. L. (2009) Platelet-derived growth factor (subtype BB) is elevated in patients with colorectal carcinoma. Diseases of the colon and rectum 52, 1166-1171.

[12] De Vita, F., Orditura, M., Lieto, E., Infusino, S., Morgillo, F., Martinelli, E., Castellano, P., Romano, C., Ciardiello, F., Catalano, G., Pignatelli, C., and Galizia, G. (2004) Elevated perioperative serum vascular endothelial growth factor levels in patients with colon carcinoma. Cancer 100, 270-278.

[13] Balkwill, F., and Mantovani, A. (2001) Inflammation and cancer: back to Virchow? Lancet 357, 539-545.

[14] Prasad, S., Gupta, S. C., and Tyagi, A. K. (2016) Reactive oxygen species (ROS) and cancer: Role of antioxidative nutraceuticals. Cancer letters.

[15] Traves, P. G., Luque, A., and Hortelano, S. (2012) Macrophages, inflammation, and tumor suppressors: ARF, a new player in the game. Mediators of inflammation 2012, 568783

[16] Yasmin, R., Siraj, S., Hassan, A., Khan, A. R., Abbasi, R., and Ahmad, N. (2015) Epigenetic regulation of inflammatory cytokines and associated genes in human malignancies. Mediators of inflammation 2015, 201703

[17] Alkim, C., Alkim, H., Koksal, A. R., Boga, S., and Sen, I. (2015) Angiogenesis in Inflammatory Bowel Disease. International journal of inflammation 2015, 970890

[18] Tas, S. W., Maracle, C. X., Balogh, E., and Szekanecz, Z. (2016) Targeting of proangiogenic signalling pathways in chronic inflammation. Nature reviews. Rheumatology 12, 111-122

[19] Valenzuela, M. A., Canales, J., Corvalan, A. H., and Quest, A. F. (2015) Helicobacter pyloriinduced inflammation and epigenetic changes during gastric carcinogenesis. World journal of gastroenterology 21, 12742-12756

[20] Mangino, G., Chiantore, M. V., Iuliano, M., Fiorucci, G., and Romeo, G. (2016) Inflammatory microenvironment and human papillomavirus-induced carcinogenesis. Cytokine \& growth factor reviews

[21] Grasso, F., and Frisan, T. (2015) Bacterial Genotoxins: Merging the DNA Damage Response into Infection Biology. Biomolecules 5, 1762-1782

[22] Darfeuille-Michaud, A., Boudeau, J., Bulois, P., Neut, C., Glasser, A. L., Barnich, N., Bringer, M. A., Swidsinski, A., Beaugerie, L., and Colombel, J. F. (2004) High prevalence of adherentinvasive Escherichia coli associated with ileal mucosa in Crohn's disease. Gastroenterology 127, $412-421$ 
[23] Mattar, M. C., Lough, D., Pishvaian, M. J., and Charabaty, A. (2011) Current management of inflammatory bowel disease and colorectal cancer. Gastrointestinal cancer research : GCR 4, 53-61

[24] Swidsinski, A., Khilkin, M., Kerjaschki, D., Schreiber, S., Ortner, M., Weber, J., and Lochs, H. (1998) Association between intraepithelial Escherichia coli and colorectal cancer. Gastroenterology 115, 281-286

[25] Arthur, J. C., Perez-Chanona, E., Muhlbauer, M., Tomkovich, S., Uronis, J. M., Fan, T. J., Campbell, B. J., Abujamel, T., Dogan, B., Rogers, A. B., Rhodes, J. M., Stintzi, A., Simpson, K. W., Hansen, J. J., Keku, T. O., Fodor, A. A., and Jobin, C. (2012) Intestinal inflammation targets cancer-inducing activity of the microbiota. Science 338, 120-123

[26] Boudeau, J., Glasser, A. L., Masseret, E., Joly, B., and Darfeuille-Michaud, A. (1999) Invasive ability of an Escherichia coli strain isolated from the ileal mucosa of a patient with Crohn's disease. Infection and immunity 67, 4499-4509

[27] Barnich, N., Carvalho, F. A., Glasser, A. L., Darcha, C., Jantscheff, P., Allez, M., Peeters, H., Bommelaer, G., Desreumaux, P., Colombel, J. F., and Darfeuille-Michaud, A. (2007) CEACAM6 acts as a receptor for adherent-invasive E. coli, supporting ileal mucosa colonization in Crohn disease. The Journal of clinical investigation 117, 1566-1574

[28] Kim, K. S., Kim, J. T., Lee, S. J., Kang, M. A., Choe, I. S., Kang, Y. H., Kim, S. Y., Yeom, Y. I., Lee, Y. H., Kim, J. H., Kim, K. H., Kim, C. N., Kim, J. W., Nam, M. S., and Lee, H. G. (2013) Overexpression and clinical significance of carcinoembryonic antigen-related cell adhesion molecule 6 in colorectal cancer. Clinica chimica acta; international journal of clinical chemistry $415,12-19$

[29] Holmer, R., Watzig, G. H., Tiwari, S., Rose-John, S., and Kalthoff, H. (2015) Interleukin-6 trans-signaling increases the expression of carcinoembryonic antigen-related cell adhesion molecules 5 and 6 in colorectal cancer cells. BMC cancer 15, 975

[30] Nougayrede, J. P., Taieb, F., De Rycke, J., and Oswald, E. (2005) Cyclomodulins: bacterial effectors that modulate the eukaryotic cell cycle. Trends in microbiology 13, 103-110

[31] Fais, T., Delmas, J., Serres, A., Bonnet, R., and Dalmasso, G. (2016) Impact of CDT Toxin on Human Diseases. Toxins 8

[32] Taieb, F., Nougayrede, J. P., Watrin, C., Samba-Louaka, A., and Oswald, E. (2006) Escherichia coli cyclomodulin Cif induces G2 arrest of the host cell cycle without activation of the DNAdamage checkpoint-signalling pathway. Cellular microbiology 8, 1910-1921

[33] Fabbri, A., Travaglione, S., and Fiorentini, C. (2010) Escherichia coli cytotoxic necrotizing factor 1 (CNF1): toxin biology, in vivo applications and therapeutic potential. Toxins 2, 283-296

[34] Balskus, E. P. (2015) Colibactin: understanding an elusive gut bacterial genotoxin. Natural product reports 32, 1534-1540

[35] Graillot, V., Dormoy, I., Dupuy, J., Shay, J. W., Huc, L., Mirey, G., and Vignard, J. (2016) Genotoxicity of Cytolethal Distending Toxin (CDT) on Isogenic Human Colorectal Cell Lines: Potential Promoting Effects for Colorectal Carcinogenesis. Frontiers in cellular and infection microbiology 6, 34

[36] Johnson, J. R., Johnston, B., Kuskowski, M. A., Nougayrede, J. P., and Oswald, E. (2008) Molecular epidemiology and phylogenetic distribution of the Escherichia coli pks genomic island. Journal of clinical microbiology 46, 3906-3911

[37] Lai, Y. C., Lin, A. C., Chiang, M. K., Dai, Y. H., Hsu, C. C., Lu, M. C., Liau, C. Y., and Chen, Y. T. (2014) Genotoxic Klebsiella pneumoniae in Taiwan. PloS one 9, e96292

[38] Nougayrede, J. P., Homburg, S., Taieb, F., Boury, M., Brzuszkiewicz, E., Gottschalk, G., Buchrieser, C., Hacker, J., Dobrindt, U., and Oswald, E. (2006) Escherichia coli induces DNA double-strand breaks in eukaryotic cells. Science 313, 848-851

[39] Putze, J., Hennequin, C., Nougayrede, J. P., Zhang, W., Homburg, S., Karch, H., Bringer, M. A., Fayolle, C., Carniel, E., Rabsch, W., Oelschlaeger, T. A., Oswald, E., Forestier, C., Hacker, J., and Dobrindt, U. (2009) Genetic structure and distribution of the colibactin genomic island among members of the family Enterobacteriaceae. Infection and immunity 77, 4696-4703 
[40] Arthur, J. C., Gharaibeh, R. Z., Muhlbauer, M., Perez-Chanona, E., Uronis, J. M., McCafferty, J., Fodor, A. A., and Jobin, C. (2014) Microbial genomic analysis reveals the essential role of inflammation in bacteria-induced colorectal cancer. Nature communications 5, 4724

[41] Baxt, L. A., Garza-Mayers, A. C., and Goldberg, M. B. (2013) Bacterial subversion of host innate immune pathways. Science 340, 697-701

[42] Mellies, J. L., and Lorenzen, E. (2014) Enterohemorrhagic Escherichia coli Virulence Gene Regulation. Microbiology spectrum 2, EHEC-0004-2013

[43] Sanchez-Villamil, J., and Navarro-Garcia, F. (2015) Role of virulence factors on host inflammatory response induced by diarrheagenic Escherichia coli pathotypes. Future microbiology 10, 1009-1033

[44] Wong Fok Lung, T., Pearson, J. S., Schuelein, R., and Hartland, E. L. (2014) The cell death response to enteropathogenic Escherichia coli infection. Cellular microbiology 16, 1736-1745

[45] Cougnoux, A., Dalmasso, G., Martinez, R., Buc, E., Delmas, J., Gibold, L., Sauvanet, P., Darcha, C., Dechelotte, P., Bonnet, M., Pezet, D., Wodrich, H., Darfeuille-Michaud, A., and Bonnet, R. (2014) Bacterial genotoxin colibactin promotes colon tumour growth by inducing a senescenceassociated secretory phenotype. Gut 63, 1932-1942

[46] Dalmasso, G., Cougnoux, A., Delmas, J., Darfeuille-Michaud, A., and Bonnet, R. (2014) The bacterial genotoxin colibactin promotes colon tumor growth by modifying the tumor microenvironment. Gut microbes 5, 675-680

[47] Cuevas-Ramos, G., Petit, C. R., Marcq, I., Boury, M., Oswald, E., and Nougayrede, J. P. (2010) Escherichia coli induces DNA damage in vivo and triggers genomic instability in mammalian cells. Proceedings of the National Academy of Sciences of the United States of America 107, 11537-11542

[48] Yates, K. E., Korbel, G. A., Shtutman, M., Roninson, I. B., and DiMaio, D. (2008) Repression of the SUMO-specific protease Senp1 induces p53-dependent premature senescence in normal human fibroblasts. Aging cell 7, 609-621

[49] Zha, L., Wilson, M. R., Brotherton, C. A., and Balskus, E. P. (2016) Characterization of Polyketide Synthase Machinery from the pks Island Facilitates Isolation of a Candidate Precolibactin. ACS chemical biology 11, 1287-1295

[50] Vizcaino, M. I., and Crawford, J. M. (2015) The colibactin warhead crosslinks DNA. Nature chemistry 7, 411-417

[51] Bossuet-Greif, N., Dubois, D., Petit, C., Tronnet, S., Martin, P., Bonnet, R., Oswald, E., and Nougayrede, J. P. (2016) Escherichia coli ClbS is a colibactin resistance protein. Molecular microbiology 99, 897-908

[52] Raisch, J., Rolhion, N., Dubois, A., Darfeuille-Michaud, A., and Bringer, M. A. (2015) Intracellular colon cancer-associated Escherichia coli promote protumoral activities of human macrophages by inducing sustained COX-2 expression. Laboratory investigation; a journal of technical methods and pathology 95, 296-307

[53] Jin, Y., Tang, S., Li, W., Ng, S. C., Chan, M. W., Sung, J. J., and Yu, J. (2016) Hemolytic E. coli Promotes Colonic Tumorigenesis in Females. Cancer research 76, 2891-2900

[54] Magdy, A., Elhadidy, M., Abd Ellatif, M. E., El Nakeeb, A., Abdallah, E., Thabet, W., Youssef, M., Khafagy, W., Morshed, M., and Farid, M. (2015) Enteropathogenic Escherichia coli (EPEC): Does it have a role in colorectal tumourigenesis? A Prospective Cohort Study. International journal of surgery 18, 169-173

[55] Li, J. P., Li, F. Y., Xu, A., Cheng, B., Tsao, S. W., Fung, M. L., and Leung, W. K. (2012) Lipopolysaccharide and hypoxia-induced HIF-1 activation in human gingival fibroblasts. Journal of periodontology 83, 816-824

[56] Mimouna, S., Goncalves, D., Barnich, N., Darfeuille-Michaud, A., Hofman, P., and VouretCraviari, V. (2011) Crohn disease-associated Escherichia coli promote gastrointestinal inflammatory disorders by activation of HIF-dependent responses. Gut microbes 2, 335-346

[57] Zhong, H., De Marzo, A. M., Laughner, E., Lim, M., Hilton, D. A., Zagzag, D., Buechler, P., Isaacs, W. B., Semenza, G. L., and Simons, J. W. (1999) Overexpression of hypoxia-inducible factor 1alpha in common human cancers and their metastases. Cancer research 59, 5830-5835 
[58] Whelan, K. A., Caldwell, S. A., Shahriari, K. S., Jackson, S. R., Franchetti, L. D., Johannes, G. J., and Reginato, M. J. (2010) Hypoxia suppression of Bim and Bmf blocks anoikis and luminal clearing during mammary morphogenesis. Molecular biology of the cell 21, 3829-3837

[59] Maddocks, O. D., Short, A. J., Donnenberg, M. S., Bader, S., and Harrison, D. J. (2009) Attaching and effacing Escherichia coli downregulate DNA mismatch repair protein in vitro and are associated with colorectal adenocarcinomas in humans. PloS one 4, e5517

[60] Ochoa, T. J., and Contreras, C. A. (2011) Enteropathogenic escherichia coli infection in children. Current opinion in infectious diseases 24, 478-483

[61] Maddocks, O. D., Scanlon, K. M., and Donnenberg, M. S. (2013) An Escherichia coli effector protein promotes host mutation via depletion of DNA mismatch repair proteins. mBio 4, e0015200113

[62] Elliott, S. J., Sperandio, V., Giron, J. A., Shin, S., Mellies, J. L., Wainwright, L., Hutcheson, S. W., McDaniel, T. K., and Kaper, J. B. (2000) The locus of enterocyte effacement (LEE)-encoded regulator controls expression of both LEE- and non-LEE-encoded virulence factors in enteropathogenic and enterohemorrhagic Escherichia coli. Infection and immunity 68, 6115-6126

[63] McNamara, B. P., and Donnenberg, M. S. (1998) A novel proline-rich protein, EspF, is secreted from enteropathogenic Escherichia coli via the type III export pathway. FEMS microbiology letters 166, 71-78

[64] Khan, A. A. (2014) In silico prediction of escherichia coli proteins targeting the host cell nucleus, with special reference to their role in colon cancer etiology. Journal of computational biology : a journal of computational molecular cell biology 21, 466-475

[65] Khan, A. A., Khan, Z., Malik, A., Shrivastava, A., Jain, S. K., and Alshamsan, A. (2015) Computational prediction of Escherichia coli proteins host subcellular targeting and their implications in colorectal cancer etiology. Cancer letters 364, 25-32

[66] Dean, P., Scott, J. A., Knox, A. A., Quitard, S., Watkins, N. J., and Kenny, B. (2010) The enteropathogenic E. coli effector EspF targets and disrupts the nucleolus by a process regulated by mitochondrial dysfunction. PLoS pathogens 6, e1000961

[67] Peralta-Ramirez, J., Hernandez, J. M., Manning-Cela, R., Luna-Munoz, J., Garcia-Tovar, C., Nougayrede, J. P., Oswald, E., and Navarro-Garcia, F. (2008) EspF Interacts with nucleationpromoting factors to recruit junctional proteins into pedestals for pedestal maturation and disruption of paracellular permeability. Infection and immunity 76, 3854-3868

[68] Choi, H. J., Kim, J., Do, K. H., Park, S. H., and Moon, Y. (2013) Enteropathogenic Escherichia coli-induced macrophage inhibitory cytokine 1 mediates cancer cell survival: an in vitro implication of infection-linked tumor dissemination. Oncogene 32, 4960-4969

[69] Roxas, J. L., Koutsouris, A., and Viswanathan, V. K. (2007) Enteropathogenic Escherichia coliinduced epidermal growth factor receptor activation contributes to physiological alterations in intestinal epithelial cells. Infection and immunity 75, 2316-2324

[70] Chen, Z., Gao, S., Wang, D., Song, D., and Feng, Y. (2016) Colorectal cancer cells are resistant to anti-EGFR monoclonal antibody through adapted autophagy. American journal of translational research 8, 1190-1196

[71] Dasari, A., and Messersmith, W. A. (2010) New strategies in colorectal cancer: biomarkers of response to epidermal growth factor receptor monoclonal antibodies and potential therapeutic targets in phosphoinositide 3-kinase and mitogen-activated protein kinase pathways. Clinical cancer research : an official journal of the American Association for Cancer Research 16, 38113818

[72] De Robertis, M., Loiacono, L., Fusilli, C., Poeta, M. L., Mazza, T., Sanchez, M., Marchionni, L., Signori, E., Lamorte, G., Vescovi, A. L., Garcia-Foncillas, J., and Fazio, V. M. (2016) Dysregulation of EGFR pathway in EphA2 cell subpopulation significantly associates with poor prognosis in colorectal cancer. Clinical cancer research : an official journal of the American Association for Cancer Research

[73] Roxas, J. L., Ryan, K., Vedantam, G., and Viswanathan, V. K. (2014) Enteropathogenic Escherichia coli dynamically regulates EGFR signaling in intestinal epithelial cells. American journal of physiology. Gastrointestinal and liver physiology 307, G374-380 
[74] El-Mosalamy, H., Salman, T. M., Ashmawey, A. M., and Osama, N. (2012) Role of chronic E. coli infection in the process of bladder cancer- an experimental study. Infectious agents and cancer 7,19

[75] Yu, H., Meng, H., Zhou, F., Ni, X., Shen, S., and Das, U. N. (2015) Urinary microbiota in patients with prostate cancer and benign prostatic hyperplasia. Archives of medical science : AMS 11, 385-394

[76] Boehm, B. J., Colopy, S. A., Jerde, T. J., Loftus, C. J., and Bushman, W. (2012) Acute bacterial inflammation of the mouse prostate. The Prostate 72, 307-317

[77] Salman, J. W., Schoots, I. G., Carlsson, S. V., Jenster, G., and Roobol, M. J. (2015) Prostate Specific Antigen as a Tumor Marker in Prostate Cancer: Biochemical and Clinical Aspects. Advances in experimental medicine and biology 867, 93-114

[78] Foxman, B. (2010) The epidemiology of urinary tract infection. Nature reviews. Urology 7, 653660

[79] Bronowski, C., Smith, S. L., Yokota, K., Corkill, J. E., Martin, H. M., Campbell, B. J., Rhodes, J. M., Hart, C. A., and Winstanley, C. (2008) A subset of mucosa-associated Escherichia coli isolates from patients with colon cancer, but not Crohn's disease, share pathogenicity islands with urinary pathogenic E. coli. Microbiology 154, 571-583

[80] Muenzner, P., Kengmo Tchoupa, A., Klauser, B., Brunner, T., Putze, J., Dobrindt, U., and Hauck, C. R. (2016) Uropathogenic E. coli Exploit CEA to Promote Colonization of the Urogenital Tract Mucosa. PLoS pathogens 12, e1005608

[81] Tolg, C., Sabha, N., Cortese, R., Panchal, T., Ahsan, A., Soliman, A., Aitken, K. J., Petronis, A., and Bagli, D. J. (2011) Uropathogenic E. coli infection provokes epigenetic downregulation of CDKN2A (p16INK4A) in uroepithelial cells. Laboratory investigation; a journal of technical methods and pathology 91, 825-836

[82] Feng, W., Han, Z., Zhu, R., Liu, P., and Liu, S. (2015) Association of p16 gene methylation with prostate cancer risk: a meta-analysis. Journal of B.U.ON. : official journal of the Balkan Union of Oncology 20, 1074-1080 